\title{
Nota Técnica: \\ Validez de los conceptos y modelos vigentes de vida de servicio de estructuras de hormigón ante los efectos del cambio climático global. Situación actual
}

\author{
Technical Note: \\ Credibility of concepts and models about service life of concrete structures \\ in the face of the effects of the global climatic change. A critical review
}

\author{
J. M. Mendoza-Rangel ${ }^{(*)}$, P. Castro-Borges ${ }^{(*)}$
}

Recepción/Received: 12-II-2008

Aceptación/Accepted: 15-IV-2008

Publicado online/Online publishing: 9-XII-09

\section{RESUMEN}

Actualmente, las definiciones de vida de servicio involucran más consideraciones que hace treinta años. Esto se debe a que en ese entonces sólo se tomaban en cuenta parámetros mecánicos. Hace algunos años se introdujeron otros parámetros como la durabilidad cuya inclusión para predecir vida de servicio y para validar el comportamiento futuro de la estructura es complicado. Esto se debe principalmente a la mezcla de variables, métodos, materiales y ambientes cuyo comportamiento es difícil de predecir. Estos factores toman especial importancia ante los efectos del cambio climático que ocasiona comportamientos ambientales impredecibles y difícilmente modelables en términos de vida útil del hormigón.

El objetivo de este trabajo es revisar críticamente las definiciones de durabilidad y vida de servicio de diversos códigos y asociaciones internacionales, haciendo énfasis en los puntos que deben mejorar para quedar más acordes a las exigencias que suponen los efectos del cambio climático. Con base en esto, se proponen nuevas definiciones de vida de servicio y durabilidad. Se propone modificar la filosofía de los enfoques de vida útil que deben hacer énfasis en dividir la vida de servicio en varias etapas para tomar en cuenta el cambio climático global.

Palabras clave: hormigón, cambio climático, durabilidad, vida de servicio, modelos de predicción.

\section{SUMMARY}

Service life definitions involve more considerations today than thirty years ago. This is because only mechanical parameters were taken into account by then. Other parameters like durability were introduced a few years ago but it is difficult to use these parameters to predict service life and validate, nowadays, future behavior. This is due mainly to a mixture of variables, methods, materials and environments whose behavior is hard to predict. These factors take special importance facing the effects from the climatic change that causes unpredictable and hardly modelling environmental behavior in terms of service life of the concrete.

The aim of this work is to review critically the definitions of durability and service life of diverse codes and international associations, making emphasis in the points that must be improved to be in accordance with the requirements that suppose the effects of the climatic change. With base in this, new definitions of service life and durability were set out. A proposal to modify the philosophy of the approaches of service life must make emphasis in dividing service life in several stages, which could be the key to take into account the global climatic change.

Key Words: concrete, climatic change, durability, service life, prediction models.

(*) Centro de Investigación y Estudios Avanzados del IPN Unidad Mérida, Mérida (Yucatán, México). 


\section{DEFINICIÓN DE CAMBIO CLIMÁTICO Y SUS CONSECUENCIAS}

Es importante definir el concepto de cambio climático y analizar las consecuencias de éste en el medio ambiente, las estructuras y, en general, en la vida del ser humano. El panel intergubernamental para el cambio climático (IPCC) (1) a través del tercer informe de evaluación "Cambio Climático 2001", define al cambio climático como: "cualquier cambio del clima a lo largo del tiempo, ya sea debido a la variabilidad natural o como consecuencia de la actividad humana". De acuerdo a este mismo reporte, este cambio en el clima ha provocado que la temperatura media de la superficie (es decir, el promedio de la temperatura del aire cerca de la superficie de la tierra y de la temperatura de la superficie del mar) haya subido desde 1861 . Durante el siglo XX, el aumento ha sido de $0,6 \pm 0,2^{\circ} \mathrm{C}$. Los datos de los satélites muestran que es muy probable (en este reporte (1) se ha utilizado la expresión "muy probable" para indicar de un 90 a $99 \%$ de probabilidades) que haya habido disminuciones de un $10 \%$ en la extensión de la capa de nieve desde finales de los años 60 . Los datos de los mareógrafos muestran que el nivel medio del mar en el mundo subió entre 0,1 y 0,2 metros durante el siglo XX. El contenido de calor mundial de los océanos ha aumentado desde finales de los años cincuenta, periodo para el que se dispone de observaciones adecuadas de las temperaturas submarinas. Es muy probable que las precipitaciones hayan aumentado de 0,5 a 1\% por decenio en el siglo XX en la mayoría de las latitudes medias y altas de los continentes del hemisferio norte y es probable que la cantidad de lluvia haya aumentado de 0,2 a 0,3\% por decenio en las regiones tropicales (de $10^{\circ} \mathrm{N}$ a $10^{\circ} \mathrm{S}$ ).

Ahora bien, los cambios en el clima se producen como consecuencia de la variabilidad interna dentro del sistema climático y de factores externos (tanto naturales como antropógenos) (1). La influencia de diversos factores externos en el clima permite ampliar comparaciones mediante el concepto de forzamiento radiativo (el forzamiento radiativo es una medida de la influencia que un factor ejerce en la modificación del equilibrio entre la energía entrante y saliente en el sistema Tierra-atmósfera, y es un índice de la importancia del factor como mecanismo potencial de cambio climático. Se expresa en vatios por metro cuadrado $\left.\left(\mathrm{Wm}^{-2}\right)\right)$. Un forzamiento radiativo positivo, como el que se produce por las crecientes concentraciones de gases de efecto invernadero, tiende a calentar la superficie. Un forzamiento radiativo negativo, que puede deberse a un aumento de ciertos tipos de aerosoles (partículas microscópicas suspendidas en el aire), tiende a enfriar la superficie.

El reporte (1) indica que la concentración atmosférica de dióxido de carbono $\left(\mathrm{CO}_{2}\right)$ ha aumentado en un $31 \%$ desde 1750. La concentración actual de $\mathrm{CO}_{2}$ no se había superado en los últimos 420.000 años y es probable que tampoco en los últimos 20 millones de años. Unas tres cuartas partes de las emisiones antropógenas de $\mathrm{CO}_{2}$ en la atmósfera durante los últimos 20 años se deben a la quema de combustibles de origen fósil. El resto se debe principalmente a cambios en el uso de la tierra, especialmente la deforestación. Los océanos y la tierra actualmente captan juntos la mitad de las emisiones antropógenas de $\mathrm{CO}_{2}$.

Fenómenos como el incremento de $\mathrm{CO}_{2}$, temperatura, niveles de los océanos, cloruros en sitios donde antes no había, etc., han afectado también a las estructuras que el ser humano ha construido, afectando de manera aleatoria su durabilidad y vida de servicio. Éstos y otros fenómenos deben ser tomados en cuenta en las definiciones de durabilidad y vida de servicio, así como en los enfoques conocidos de vida de servicio.

\section{DEFINICIONES ACTUALES DE DURABILIDAD FRENTE AL CAMBIO CLIMÁTICO}

En la Tabla 1 se muestran varias definiciones de durabilidad procedentes de diferentes asociaciones e instituciones. Estándares como los japoneses (AIJ 1993) (2) y la Sociedad Japonesa de Ingenieros Civiles (JSCE 1995) (3), la del Reino Unido (PD 6534:1993)(4) y el canadiense (CSA 47895) (5) mencionan a la durabilidad como parte importante en el diseño de estructuras, sin embargo no dan una definición específica que la describa claramente, dejando abierta la posibilidad de que los usuarios de las normativas la interpreten de diferente manera y ello afecte no solo a los proyectos sino a las predicciones de vida de servicio.

Otros códigos e instituciones definen en forma explícita el término "durabilidad", pero, como es natural, las restricciones en cuanto a lenguaje, semántica y costumbres de cada lugar, también ocasionan interpretaciones diferentes con efectos similares a los mencionados arriba. Aunque muy completa, la definición de durabilidad dada en la norma NMX-C-403-ONNCCE-1999 (6) sólo se refiere al comportamiento del hormigón en sí mismo y no presenta información sobre la durabilidad en las estructuras. Ahondar en las diferentes interpretaciones de durabilidad para un material, elemento o estructura, podría ser de mucha utilidad para esta norma. Por su parte, la instrucción española EHE (7), sí considera el concepto de durabilidad para la estructura completa y especifica su validez para la vida de servicio proyectada. Sin embargo, aunque contempla los efectos diferentes a los ocasionados por las cargas contempladas en el análisis estructural, definirlos en futuras versiones permitiría, no solo una versión más completa, sino también un mejor entendimiento y aplicación de la misma. Por otro lado la norma americana ACI 365.1R-00 (8) hace referencia a productos, componentes y construcción. La ACI 365 define a la durabilidad como una "habilidad" y éste es un término muy importante. A pesar de ser muy interesante y aplicable, la definición de 
la ACI necesita referirse con precisión a las condiciones ambientales y a los probables cambios de uso de la estructura. Los tres documentos, ACI, EHE y NMX necesitan incluir parámetros como los cambios de uso y posibles vicios ocultos que promueven las fallas después de la puesta en servicio de la estructura. De la misma Tabla 1, la norma brasileña NBR 6118 (9) menciona específicamente a las influencias ambientales previstas y definidas por el proyectista y el contratista desde la concepción del proyecto, siendo también muy importante que en futuras versiones las defina para promover un mejor conocimiento del medio técnico y una mejor inclusión del parámetro "durabilidad" en los proyectos.

La Organización Europea para Aprobaciones Técnicas (EOTA) (10), a través del reporte técnico "Characterisation, Aspects of Durability and Factory Production Control for Reactive Materials, Components and Products", contempla un apartado de durabilidad de componentes o materiales reactivos, sin definir la primera explícitamente, mas si ofrecen pruebas y ensayos para determinarla en dichos materiales o componentes. Los casos de organismos y normas que describen en forma implícita a la durabilidad propician interpretaciones muy amplias cuyos resultados al momento de hacer un proyecto o evaluar una estructura podrían no ser representativos del sitio y las condiciones climáticas en los que se aplique.

Así como estas normas, por lo general, cualquiera de las que se pudiese consultar, podría tener su propia descripción de durabilidad, ya sea explícita o implícita. Sin embargo, diferencias tan pequeñas entre ellas como las descritas arriba, así como las diferentes formas en las que podemos interpretar su significado, dificultan el uso explícito del término "durabilidad" para realizar predicciones de vida de servicio universales.

Por otra parte, en sus definiciones, las normas actuales no definen las acciones ambientales que no solo son variadas, sino que están actualmente afectadas por el cambio climático global, el cual sugiere tener cuidado y previsión al definir éstas al momento de elaborar un proyecto estructural.

Las Asociaciones e Instituciones de la Tabla 1 han promovido en forma pionera las definiciones de durabilidad descritas arriba. Sin embargo, los últimos acontecimientos relacionados con el clima global hacen necesaria su adecuación a esta situación.

Tomando como base esta discusión y las limitaciones de los términos actuales, una definición acorde con la realidad actual, el cambio climático y que retoma lo mejor de lo expresado en la bibliografía podría ser:

\section{DEFINICIÓN DE DURABILIDAD PROPUESTA QUE TOMA EN CUENTA EL EFECTO DEL CAMBIO CLIMÁTICO GLOBAL}

Capacidad de un material de construcción, elemento o estructura de hormigón de resistir las acciones físicas, químicas, biológicas y ambientales vinculadas al efecto del cambio climático global con su entorno durante un

Tabla 1

Definiciones de Durabilidad.

\begin{tabular}{|c|c|c|}
\hline Código o Institución & Definición de durabilidad & Año \\
\hline Instituto de Arquitectos de Japón (AIJ) (2) & No la define explícitamente. & 1993 \\
\hline $\begin{array}{l}\text { Guide to the use in the UK of DD ENV 206: } 1992 \text { Concrete. (PD } \\
\qquad 6534: 1993)(4)\end{array}$ & No la define explícitamente. & 1993 \\
\hline Japan Society of Civil Engineers (JSCE 1995) (3) & No la define explícitamente. & 1995 \\
\hline Canadian Standard CSA 478-95 (5) & No la define explícitamente. & 1995 \\
\hline Norma Mexicana NMX-C-403-ONNCCE-1999 (6) & $\begin{array}{c}\text { Es la capacidad del hormigón hidráulico para uso estructural de resistir } \\
\text { durante un tiempo determinado la acción ambiental, ataque químico, } \\
\text { abrasión, corrosión del acero de refuerzo o cualquier otro proceso de } \\
\text { deterioro para mantener su forma original, condición de servicio } \\
\text { y propiedades mecánicas. }\end{array}$ & 1999 \\
\hline Instrucción Española de Hormigón Estructural EHE (7) & $\begin{array}{l}\text { La durabilidad de una estructura de hormigón es su capacidad } \\
\text { para soportar, durante la vida útil para la que ha sido proyectada, } \\
\text { las condiciones físicas y químicas a las que está expuesta, y que } \\
\text { podrían llegar a provocar su degradación como consecuencia de } \\
\text { efectos diferentes a las cargas y solicitaciones consideradas en el } \\
\text { análisis estructural. }\end{array}$ & 1999 \\
\hline ACI 365.1R-00 (ASTM E 632) (8) & $\begin{array}{c}\text { La capacidad de mantener la serviceabilidad } \\
\text { de un producto, componente, ensamble o construcción durante un } \\
\text { tiempo especificado. }\end{array}$ & 2000 \\
\hline NBR 6118, Proyecto de estructuras de hormigón-procedimiento (9) & $\begin{array}{c}\text { Consiste en la capacidad de la estructura de resistir las influencias } \\
\text { ambientales previstas y definidas en conjunto por el autor del } \\
\text { proyecto estructural y el contratista desde el inicio de los trabajos } \\
\text { de elaboración del proyecto. }\end{array}$ & 2002 \\
\hline EOTA (10) & No la define explícitamente. & 2006 \\
\hline
\end{tabular}


tiempo determinado previsto desde el proyecto, manteniendo su serviceabilidad y conservando su forma original, propiedades mecánicas y condiciones de servicio.

Se entiende por serviceabilidad (8) como la capacidad de un producto, componente, ensamble o construcción para desempeñar las funciones para las cuales son diseñadas y construidas.

Ahora bien, esta definición debe ir acompañada, en los códigos respectivos, de la enunciación de las acciones físicas, químicas, mecánicas, biológicas y ambientales que inciden directamente en las estructuras afectando su durabilidad. Con base en lo expresado por varias publicaciones $(6,8,11-14)$ estas acciones pueden resumirse en la Tabla 2.

\section{DEFINICIONES ACTUALES DE VIDA DE SERVICIO FRENTE AL CAMBIO CLIMÁTICO}

El término durabilidad es importante para definir vida de servicio. Sin embargo, los estándares y códigos también tienen diferentes definiciones de vida de servicio y toman en cuenta diferentes enfoques que hacen de ellas más difíciles de usar como herramientas de predicción. La Tabla 3 muestra algunas definiciones: la Red DURAR (15) incluye en su definición la mayoría de las variables actuales, tales como seguridad, funcionalidad y estética. En esta definición se establece que la vida de servicio es un periodo de tiempo sin acciones de mantenimiento inesperadas. Sin embargo, la probabilidad de que las acciones de mantenimiento inesperado aumenten es mayor con los efectos, cada vez más vertiginosos, del cambio climático. Por lo tanto, dicho periodo de tiempo se acorta. Una definición de "acciones de mantenimiento inesperados" donde se incluyan los efectos del cambio climático podría darle al proyectista una idea más clara y concisa cuando elabora el proyecto.

Más tarde, The Construction Products Directive (16) especifica que la vida de servicio es un periodo de tiempo donde la estructura mantiene sus requerimientos esenciales. Estos requerimientos son: resistencia mecánica y estabilidad, seguridad en caso de fuego, higiene, salud y medio ambiente, seguridad en uso, protección contra ruido y ahorro de energía y retención de calor. Aunque muy completa, esta definición necesita contemplar lo relativo al mantenimiento de las estructuras, que resulta de vital importancia para mantener la vida útil prevista desde el proyecto ante los embates climáticos y de desgaste propios de los materiales debido al uso que se le da durante su vida de servicio.

El ACI 365.1R-00 (8) especifica que la vida de servicio es un periodo donde todas las propiedades exceden el mínimo aceptable con mantenimiento rutinario. Una especificación de las propiedades de las estructuras o componentes, que bien podrían ser físicas, químicas y mecánicas, completaría la definición. Adicionalmente, mantenimiento rutinario puede ser interpretado de diferentes maneras dependiendo del lugar donde se aplique, por lo que "mantenimiento mínimo necesario" para mantener las propiedades mínimas aceptables, se propone como una alternativa para completar la definición.

Tabla 2

Acciones físicas, químicas, mecánicas, biológicas y ambientales que inciden diractamente en las estructuras.

\begin{tabular}{|c|c|c|c|c|}
\hline Físicas $(8,11,12)$ & Químicas $(8,11,12)$ & Mecánicas (13) & Biológicas (12) & Ambientales $(6,14)$ \\
\hline Radiación térmica & Sulfatos & Tracción axial & Microorganismos & Ciclos de hielo y deshielo \\
\hline Radiación nuclear & Sales de magnesio y amonio & Compresión axial & Hongos & $\begin{array}{l}\text { Derretimiento de capas } \\
\text { subterráneas de hielo }\end{array}$ \\
\hline Fuego & Ataque ácido y básico & Flexión y Corte & Bacterias & Viento \\
\hline Humedad & Lixiviación por aguas puras & Flexión compuesta & & Lluvias \\
\hline Aerosoles & Efecto de agua de mar & Torsión & & Inundaciones \\
\hline Sales & Reacción álcali-agregado & Impacto & & Tormentas \\
\hline Ácidos & Ataque de $\mathrm{CO}_{2}$ (Carbonatación) & & & Erosión costera \\
\hline Álcalis & Ataque de cloruros & & & Huracanes \\
\hline Abrasión & & & & Radiación solar \\
\hline Erosión & & & & Temperaturas elevadas \\
\hline \multicolumn{5}{|l|}{ Cavitación } \\
\hline Agua & & & & Temperaturas bajas \\
\hline Sismos & & & & Ambientes secos \\
\hline $\begin{array}{l}\text { Movimientos por vehículos } \\
\text { o instalaciones }\end{array}$ & & & & $\begin{array}{l}\text { Ambiente húmedo sin } \\
\text { congelamiento }\end{array}$ \\
\hline Ciclos de mojado y secado & & & & $\begin{array}{l}\text { Ambiente húmedo con } \\
\text { congelamiento y agentes } \\
\text { descongelantes }\end{array}$ \\
\hline Cristalización salina. & & & & Ambiente marino \\
\hline Efectos combinados & & & & \\
\hline
\end{tabular}


Por su parte, la Instrucción Española (EHE) (7) define a la vida de servicio de una estructura como el periodo de tiempo, a partir de la fecha en la que finaliza su ejecución, durante el que deben mantenerse las exigencias básicas en unos límites aceptables. Durante ese periodo requerirá una conservación normal, que no implique operaciones de rehabilitación. La instrucción si define las exigencias básicas, sin embargo una especificación de lo que es una "conservación normal" daría una visión más precisa puesto que, lo que para unos proyectistas es normal, para otros no lo es.

El CIB W 80 (17) considera que la vida de servicio termina cuando no es posible completar la función prevista. Establecer si esa función prevista es la que se tiene desde el proyecto, o de su construcción o desde su puesta en marcha, haría de ésta definición más precisa. Por otro lado sería deseable que la definición contemplara cuáles son las funciones previstas, ya que éstas pueden ser varias y no necesariamente en el orden de importancia para uno $u$ otro proyectista. Por ejemplo, para alguien la función prevista puede ser estética y para otro puede ser la seguridad.

Existen muchos otros estándares y códigos y el factor común en ellos es que la vida de servicio se considera como un "periodo de tiempo". La verdad es que frases como "requerimientos esenciales", "acciones de mantenimiento inesperado", "mantenimiento rutinario" y "funciones que no se cumplen" son importantes, pero pueden ser interpretadas y usadas de muchas maneras. Consecuentemente, uno debe ser muy cuidadoso cuando utilice estas definiciones en modelos para predecir durabilidad. Es necesario incluir más guías específicas cuando se usen definiciones de vida de servicio. De acuerdo a lo discutido hasta aquí, una definición de vida de servicio que tome en cuenta los efectos del cambio climático, retomando lo mejor de las acepciones actuales puede ser:

\section{DEFINICIÓN PROPUESTA DE VIDA DE SERVICIO QUE TOMA EN CUENTA EL EFECTO DEL CAMBIO CLIMÁTICO GLOBAL}

Es el periodo de tiempo durante el cual el desempeño de un material, elemento o estructura de hormigón conserva los requerimientos de proyecto en términos de seguridad (resistencia mecánica y estabilidad, seguridad en caso de fuego, seguridad en uso), funcionalidad (higiene, salud y medio ambiente, protección contra el ruido y ahorro energético y confort térmico) y estéticos (deformaciones, agrietamientos, desconchamientos), con un mínimo de mantenimiento que permita controlar los efectos del cambio climático global en su entorno.

\section{CONTRIBUCIONES DE MODELOS DETERMINÍSTICOS Y PROBABILÍSTICOS ASÍ COMO ENFOQUES SOBRE VIDA DE SERVICIO FRENTE AL CAMBIO CLIMÁTICO GLOBAL}

Es indudable que teniendo definiciones más completas de durabilidad y vida de servicio acordes a la situación geográfica y condición climática de cada sitio, los modelos sean más precisos en sus predicciones. De aquí la importancia de realizar la siguiente revisión sobre las contribuciones de los modelos y enfoques de vida de servicio discutiendo la tendencia hacia la incorporación de variables de tiempo y clima asociadas al cambio climático.

Las Tablas 4 y 5 muestran una lista corta de contribuciones de diferentes autores que están trabajando con modelos determinísticos y probabilísticos así como en enfoques sobre vida de servicio que toman en cuenta diferentes variables en sus modelos.

Tabla 3

Definiciones de vida de servicio.

\begin{tabular}{|c|c|c|}
\hline \multirow{2}{*}{ Código o Institución } & Definición & \multirow{2}{*}{ Año } \\
\hline & Vida de Servicio & \\
\hline Red DURAR (15) & $\begin{array}{l}\text { Es el periodo de tiempo durante el cual la estructura conserva los } \\
\text { requerimientos de proyecto en términos de seguridad, funcionalidad } \\
\text { y estética, sin costos inesperados de mantenimiento. }\end{array}$ & 1997 \\
\hline Construction Products Directive (CPD) (16) & $\begin{array}{l}\text { Es el periodo de tiempo durante el cual el desempeño de los } \\
\text { trabajos estará manteniéndose a un nivel compatible con el } \\
\text { cumplimento de los requerimientos esenciales. }\end{array}$ & 1998 \\
\hline Instrucción Española de Hormigón Estructural EHE (7) & $\begin{array}{l}\text { Se entiende por vida útil de la estructura el período de tiempo, } \\
\text { a partir de la fecha en la que finaliza su ejecución, durante el que } \\
\text { deben mantenerse las exigencias básicas en unos límites } \\
\text { aceptables. Durante ese período requerirá una conservación } \\
\text { normal, que no implique operaciones de rehabilitación. }\end{array}$ & 1999 \\
\hline ACI 365.1R-00 (ASTM E 632) (8) & $\begin{array}{l}\text { Es el periodo de tiempo después de la instalación, durante el cual } \\
\text { todas las propiedades exceden los valores mínimos aceptables con } \\
\text { mantenimiento rutinario. }\end{array}$ & 2000 \\
\hline CIB W080/RILEM 175 SLM. (17) & $\begin{array}{l}\text { En general, el fin de la vida de servicio es el punto en el tiempo, } \\
\text { cuando la función prevista no se cumple. }\end{array}$ & 2004 \\
\hline Compliance Document for New Zealand Building Code (18) & No se define explícitamente. & 2004 \\
\hline
\end{tabular}


De la Tabla 4 se observa que los modelos determinísticos, debido a la poca información de datos estadísticos, fueron muy utilizados en los primeros años, ya que solo se basaban en la experiencia en campo de los proyectistas. Por lo tanto, su utilidad para predecir la vida de servicio de las estructuras estaba limitado, más aún, si consideramos que los cambios en el clima en el mundo se han acelerado en los últimos años y por ende, eran difíciles de tomar en cuenta para ese entonces. Con el paso del tiempo, conforme pasaron los años, se fueron recopilando más datos estadísticos e incorporando nuevas variables mediante ensayos en laboratorios, cuyos resultados fueron incorporados a modelos probabilísticos que resultan ser más precisos que los determinísticos en sus predicciones de vida de servicio. Sin embargo, a pesar de los buenos resultados dados por los modelos probabilísticos, éstos tienen la dificultad de no poder incorporar todos los fenómenos que intervienen y que afectan a las estructuras. Esto es una tarea por demás difícil, ya que estos fenómenos varían dependiendo de la situación geográfica y evolucionan a través del tiempo.

Derivado de la dificultad de predecir vida de servicio a través de los modelos determinísticos y probabilísticos, en los últimos años se ha visto una tendencia hacia nuevos enfoques de vida de servicio que sin duda orientarán la forma en que se desarrollen los primeros.

De las contribuciones de la Tabla 5, puede observarse que efectivamente hay una evolución a través de los modelos probabilísticos que incluyen más variables que antes y que además actúan de manera simultánea sobre las estructuras. Algunos modelos le dan mayor importancia a ciertos estados de las estructuras que otros (grietas, por ejemplo). También se observa la tendencia a modelar el comportamiento de la estructura completa en lugar de elementos aislados, y conforme el tiempo pasa, el uso de herramientas matemáticas más sofisticadas para predecir la vida de servicio a través de poderosos programas computacionales. Algunos enfoques de vida de servicio (22) han propuesto incluir una etapa que tome en cuenta un factor de seguridad en la predicción de aquélla.

Sin embargo, debido a los efectos del cambio climático, cada vez resulta más difícil predecir en su totalidad la vida de servicio de las estructuras, ya que sería complicado incluir en un modelo, los cambios en el medio ambiente que está en constante evolución $y$, por tal motivo, las afectaciones sobre las estructuras serán diferentes a través del tiempo.

La verificación de cualquier predicción bajo estas circunstancias es difícil debido a los muchos y diferentes modelos existentes, a las diferentes interpretaciones de los conceptos en estándares y códigos, a la cantidad de mezclas de viejas y nuevas variables, a los nuevos materiales y métodos de diseño, así como los cambios que se dan en el medio ambiente, etc.

Debido a este problema, autores han señalado (33) la necesidad de utilizar nuevos enfoques de vida de servicio, que dividan a la vida de servicio en varias etapas, para cada una de las cuales debe haber un tratamiento matemático diferente que permita predecir su comportamiento en un espacio limitado de tiempo. Este enfoque permitiría realizar predicciones por etapa, que contemple solo los fenómenos que intervienen en dicha etapa, como, por ejemplo, los del medio ambiente. La restricción de las predicciones al contorno de condiciones climáticas es ya un tema que recientemente se maneja (34) en la literatura al

Tabla 4

Autores trabajando en modelos determinísticos, probabilísticos y enfoques esquemáticos.

\begin{tabular}{|c|c|c|c|c|}
\hline Autor & Modelos determinísticos & Modelos probabilísticos & Enfoques esquemáticos & Año \\
\hline Tuutti (19) & & & $\mathrm{x}$ & 1982 \\
\hline Masters (20) & $\mathrm{x}$ & & & 1987 \\
\hline British Standard Institution (21) & $x$ & & & 1992 \\
\hline C. Andrade (22) & & & $\mathrm{x}$ & 1994 \\
\hline Canadian Standard Association (5) & $\mathrm{x}$ & & & 1995 \\
\hline Sarja and Vesikari (23) & $\mathrm{x}$ & $\mathrm{x}$ & & 1996 \\
\hline K. Pettersson (24) & & & $x$ & 1998 \\
\hline R. de Coss, G. Murrieta, P. Castro (25) & & $\mathrm{x}$ & & 1998 \\
\hline Paulo Helene (26) & & & $\mathrm{x}$ & 1998 \\
\hline Siemes, T., Edvarsen, C. (27) & & $\mathrm{x}$ & & 1999 \\
\hline S. Caré and E. Hervé (28) & $x$ & & & 2000 \\
\hline Siemes, T., de Vries, H. (29) & & & & 2002 \\
\hline Steen Rostam (30) & & $\mathrm{x}$ & $x$ & 2003 \\
\hline CIB W80/RILEM 175 SLM (17) & & & & 2004 \\
\hline A. Lindvall (31) & & $x$ & & 2005 \\
\hline Jieying Zhang, Zoubir Lounis (32) & & $x$ & $x$ & 2006 \\
\hline P. Castro-Borges and P. Helene (33) & & & $x$ & 2007 \\
\hline
\end{tabular}


Validez de los conceptos y modelos vigentes de vida de servicio de estructuras de hormigón ante los efectos del cambio climático global. Situación actual Credibility of concepts and models about service life of concrete structures in the face of the effects of the global climatic change. A critical review

Tabla 5

Principales contribuciones de diferentes autores y códigos.

\begin{tabular}{|c|c|c|}
\hline Autor & Contribución & Año \\
\hline Tuutti (19) & Modelo esquemático (enfoque de vida de servicio) con periodos de iniciación y propagación. & 1982 \\
\hline Masters (20) & Sistema de requerimientos para la predicción de vida de servicio. & 1987 \\
\hline British Standard Institution (21) & $\begin{array}{l}\text { La predicción de vida de servicio de una construcción deberá ser evaluada por: referencias a } \\
\text { experiencias previas con construcciones iguales o similares; por interpolación de pruebas aceleradas. }\end{array}$ & 1992 \\
\hline C. Andrade (22) & Modelo esquemático que incluye una etapa con factor de seguridad. & 1994 \\
\hline Canadian Standard Association (5) & $\begin{array}{c}\text { Predicción de vida de servicio asumiendo condiciones ambientales e instalaciones, } \\
\text { procedimiemtos de operación y mantenimiento. }\end{array}$ & 1995 \\
\hline Sarja and Vesikari (23) & $\begin{array}{c}\text { Degradación, desempeño, vida de servicio, y modelos de durabilidad determinísticos } \\
\text { y estocásticos. }\end{array}$ & 1996 \\
\hline K. Pettersson (24) & $\begin{array}{l}\text { Vida de servicio por corrosión inducida por cloruros en hormigones fracturados de alta } \\
\text { resistencia. }\end{array}$ & 1998 \\
\hline R. de Coss, G. Murrieta, P. Castro (25) & Efecto de los ciclos climáticos sobre la difusión de cloruros en hormigones porosos. & 1998 \\
\hline Paulo Helene (26) & Predicción de vida de servicio mediante un modelo esquemático con nuevas etapas. & 1998 \\
\hline Siemes, T., Edvarsen, C. (27) & $\begin{array}{l}\text { Método probabilístico de predicción del comportamiento de las estructuras de hormigón. } \\
\text { Define cuatro etapas: despasivación, fractura, desconchamiento y colapso. }\end{array}$ & 1999 \\
\hline S. Caré and E. Hervé (28) & $\begin{array}{l}\text { Predicción del coeficiente de difusión de cloruros en hormigones basado en las proporciones } \\
\text { de la mezcla. }\end{array}$ & 2000 \\
\hline Siemes, T., de Vries, H. (29) & $\begin{array}{c}\text { Modelo refinado de cinco etapas para el hormigón: despasivación, fractura, desconchamiento, } \\
\text { pérdida de adhererncia y colapso. }\end{array}$ & 2002 \\
\hline Steen Rostam (30) & $\begin{array}{l}\text { Modelar los mecanismos de deterioración, es necesario el tratamiento probabilistico } \\
\text { de incertidumbres, la vida de servicio afecta a todas las partes involucradas. }\end{array}$ & 2003 \\
\hline CIB W80/RILEM 175 SLM (17) & Modelos sugeridos, entre ellos, modelos determinísticos o método de los factores. & 2004 \\
\hline A. Lindvall (31) & Modelos para acciones ambientales sobre estructuras de hormigón reforzado. & 2005 \\
\hline Jieying Zhang, Zoubir Lounis (32) & $\begin{array}{l}\text { Análisis sensitivo de un modelo simplificado de iniciación de corrosión basado en la difusión } \\
\text { en estructuras de hormigón expuestas a cloruros. }\end{array}$ & 2006 \\
\hline P. Castro-Borges and P. Helene (33) & $\begin{array}{c}\text { Enfoque de vida de servicio a través de un nuevo modelo esquemático que toma en cuenta } \\
\text { más etapas. }\end{array}$ & 2007 \\
\hline V. Baroghel-Bouny, Thai Luang Nguyen (34) & Enfoque que propone modelo multi-nivel. & 2008 \\
\hline
\end{tabular}

momento de aplicar métodos de predicción de vida de servicio como el de los indicadores.

\section{CONCLUSIÓN}

De acuerdo con la literatura consultada, se puede concluir que es necesario mejorar las definiciones actuales de vida de servicio y durabilidad de varios códigos y estándares. Se propusieron en este artículo nuevas definiciones de vida de servicio y durabilidad que toman en cuenta variables asociadas al cambio climático global. Se discutió brevemente la tendencia de aportación de los modelos de vida de servicio haciendo ver la necesidad de trabajar también en nuevos enfoques de vida de servicio que tomen en cuenta etapas bien definidas de vida de servicio. Esto permitiría mejores predicciones de vida de servicio con los modelos actuales.

\section{AGRADECIMIENTOS}

Uno de los autores, J.M. Mendoza-Rangel agradece la beca doctoral de CONACYT. Este trabajo es parcialmente financiado por CINVESTAV y CONACYT (Proyectos J200.814, J200.653, CIAM 54826, SEP 57420). El punto de vista de este trabajo es de los autores y no necesariamente de las organizaciones que lo financian.

\section{BIBLIOGRAFÍA}

(1) The intergovernmental panel of climate change (IPCC): "Thirth Evaluation Inform, Climatic Change 2001, The Scientific Base" (2001).

(2) Architectural Institute of Japan (AIJ): "The English Edition of Principal Guide for Service Life Planning of Buildings", Architectural Institute of Japan, Japan (1993).

(3) Japan Society of Civil Engineers (JSCE): "Proposed Specification Of Durability Design For Concrete Structures", Translation from the Concrete Library No. 82 published by JSCE, Japan (1995).

(4) Published Document PD 6534:1993: "Guide to the use in the UK of DD ENV 206: 1992 Concrete. Performance, production, placing and complance criteria", United Kingdom (1993). 
(5) Canadian Standars Association (CSA S478-1995): "Guideline on durability in buildings", Canada (1995).

(6) NMX-C-403-ONNCCE-1999: "Construction Industry - Hydraulic Concrete for Structural Use" (in Spanish), México (1999).

(7) Spanish Instruction of Structural Concrete (EHE), Chapter VII, B.O.E. January 13th (1999).

(8) American Concrete Institute (ACI) 365.1R-00: "Service-Life Prediction, State-of-the-Art Report", reported by ACI Committee 365 (2000).

(9) Brazilian Association of Technical Codes, NBR 6118: "Concrete Structures Project-Procedure" (2002).

(10) European Organization for Technical Approvals (EOTA): "Characterisation, Aspects of durability and Factory Production Control for Reactive Materials, Components and Products", TR024 (2006).

(11) Lifecon Deliverable D3.2: "Service Life Models, Instructions on methodology and application of models for the prediction of the residual service life for classified environmental loads and types of structures in Europe", Life Cycle Management of Concrete Infrastructures for Improved Sustainability, Lay, Sascha and Schießl, Peter, authors (2003).

(12) Sanjuán Barbudo, M. A.; Castro-Borges, P.: "Action of the Chemical and Phisical agents over Concrete", Mexican Institute of Cement and Concrete (IMCYC), ISBN 968-464-097-8 (2001), pp. 3-27.

(13) Manual of Concrete Structures Rehabilitation: "Raparation, Reinforcement and protection", Helene, P. And Pereira F., Editors, ISBN 85903707-1-2 (2003).

(14) Canadian Standards Assosiation (CSA): "The Role of Standards in Adapting Canada's Infrastructure to the Impacts of Climate Change" (2006).

(15) DURAR Network: "Inspection, evaluation and Diagnostic manual of Corrosion in Reinforced Concrete Structures", CYTED, Iberoamerican Program of Science and Tecnology for Development, Subprogram XV Environmental Corrosion/Impact about Materials, Maracaibo, Venezuela, CYTED (1997).

(16) Construction Products Directive (CPD), European Community Council 89/106/EWG updated 93/68/EWG (1998).

(17) CIB W80 / RILEM 175 SLM: "Service Life Methodologies Prediction of Service Life for Building and Components", Task Group Performance Based Methods for Service Life Prediction, State of the Art Reports, March (2004).

(18) New Zealand Building Code, Clause B2: "Durability", ISBN 0-477-01606-5 (2004).

(19) Tuutti, K.: "Corrosion of steel in concrete", CBI, Research report 4, 1982, Stockholm (1982).

(20) Masters, L. W.: "Service life prediction - A state of the art", $4^{\text {th }}$ International Conference on Durabilty of Building Materials and Components, Singapore, 4-6 November (1987).

(21) British Standards Institution: BS 7543:1992 Guide to Durability of Buildings and Building Elements, Products and Components. British Standards Institution, London, UK (1992).

(22) Andrade, C.: "Quantification of durability of reinforcing steel, methods and calculation procedures". Of Concrete Technology: New Trends, Industrial Applications. Edited by A. Aguado, R. Gettu and S. P. Shah. Rilem. Published by E\&FN Spon, 2-6 Boundary Row, London SE1 8HN, UK ISBN 041920150 5, pp. 158-175 (1994).

(23) Sarja, A.; Vesikari, E.: "Durability Design of Concrete Structures", Manuscript of RILEM Report of TC 130-CSL, RILEM Report Series 14, Chapter 7 Durability models, pp. 97-111, E \& FN Spon, Chapman and Hall, 165 p (1996).

(24) Pettersson, K.: "Service life of concrete structure including the propagation time", Concrete Under Severe Conditions 2/1998, O. E. Gjorv, K. Sakai, N. Banthia (editors), vol. 1, p. 489, International Conference on Concrete Under Severe Condition, Norway (1998).

(25) De Coss, R.; Murrieta, G.; Castro, P.: "Effect of weather cycles on chloride diffusion in porous concrete", in Rehabilitation of Corrosion Damaged Infrastructure, Chapter IV: Modeling, methods, techniques and technologies. August, 1998, P. Castro, O. Troconis, C. Andrade (editors), NACE International, ISBN-970-92095-0-7 (1998), pp. 285-293.

(26) Helene, P.: "Service life of concrete structures", in VI congress of quality central (CONPAT 1997), University of Rio Grande del Sur, vol. 1 (1997), pp. 1-30.

(27) Siemes, T.; Edvardsen, C.: "DuraCrete: Service life design for concrete structures", 8DBMC, (1999), pp. 1343-1356.

(28) Caré, S.; Hervé, E.: "Prediction of the chloride diffusion coefficient in concrete using the homogenzation technique", in Second International RILEM Workshop on Testing and Modeling the Chloride Ingress into Concrete, C. Andrade, J. Kropp (editors), Paris, France (2000).

(29) Siemes, T.; Vries, H.: "Overview of the development of service life design for concrete structures", 9DBMC, paper 261 (2002).

(30) Rostam, S.: "Service life of concrete structures - a design approach for the future", in Concreto Colloquia, São Carlos, SP, Brazil (2003).

(31) Lindvall, A.: "Models for environmental actions on reinforced concrete structures", in Repair and Renovation of Concrete Structures, Ravindra K Dhir, M. Roderick Jones, and Li Zheng (editors), International Conference Held, at the University of Dundee, Scotland, UK (2005), pp. 65-72.

(32) Zhang, J.; Lounis, Z.: "Sensitivity analysis of simplified difusion-based corrosion initiation model of concrete structures exposed to chlorides", in Cement and Concrete Research, 36, 1312-1323 (2006).

(33) Castro-Borges, P.; Helene, P.: "Service Life of Reinforced Concrete Structures. New Approach", ECS Transactions, vol. 3, no. 13, ISBN 978-1-56677-540-3, pp. 9-14 (2007).

(34) Baroghel-Bouny, V.; Thai Luang Nguyen: "Performance-based design of concrete and multi-level prediction of RC durability in coastal and marine environments", MEDACHS08, Construction Heritage in Coastal and Marine Environments, Damage, diagnostics, maintenance, and rehabilitation. Lisbon, Portugal, 28-29 January (2008). 